\title{
MORFOMETRIA DE PUPAS E ADULTOS DE Rhinochenus stigma LINNAEUS 1758 (COLEOPTERA: CURCULIONIDAE) ${ }^{1}$
}

\author{
Arley José Fonseca², Claubert Wagner Guimarães de Menezes², Conceição Aparecida dos Santos³, \\ Sebastião Lourenço de Assis Júnior ${ }^{4}$, Adriano Geraldo Fonseca ${ }^{4}$ e Marcus Alvarenga Soares ${ }^{4}$
}

\begin{abstract}
RESUMO - Besouros do gênero Rhinochenus sp. (Coleoptera: Curculionidae) danificam sementes de espécies da flora do Cerrado brasileiro como o jatobá (Hymenea courbaril L.) e a copaíba (Copaifera langsdorffii Desf.). Este trabalho teve como objetivo avaliar a morfometria de pupas e adultos de $R$. stigma, bem como aspectos comportamentais, como a emissão de sons, para auxiliar a sexagem desta espécie. Os insetos foram obtidos de frutos de jatobá coletados no Município de São Gonçalo do Rio Preto, em Minas Gerais, Brasil. As características morfométricas de pupas e adultos não apresentaram diferenças pelo teste t, não sendo seguras para a diferenciação dos sexos. Para adultos de $R$. stigma, a sexagem só foi possível após a morte, pela dissecação dos órgãos genitais.

Palavras-chave: Cerrado; Praga florestal; Sexagem.

\section{MORPHOMETRY OF PUPAE AND ADULTS OF THE Rhinochenus stigma LINNAEUS 1758 (COLEOPTERA: CURCULIONIDAE)}

\begin{abstract}
Beetles of genus Rhinochenus sp. (Coleoptera: Curculionidae) damage the seeds of species of flora of Brazilian savannah such as jatobá (Hymenea courbaril L.) and copaiba (Copaifera langsdorffii Desf.). Thus, the objective of the present was to evaluate the morphology of pupae and adult $\boldsymbol{R}$. stigma, as well as behavioral aspects such as emission of sounds, to assist in sexing this species. The insects were obtained from jatobá fruits collected in São Gonçalo do Rio Preto, Minas Gerais State, Brazil. The morphometric characteristics of both phases did not differ by t test, being not safe for the differentiation of the sexes. For adults of R. stigma, sexing was possible only after death, by dissection of the genitalia.
\end{abstract}

Keywords: Forest pest; Savannah; Sexing.

\section{INTRODUÇÃO}

O bioma Cerrado brasileiro compreende extensa área de cerca de dois milhões de $\mathrm{km}^{2}$ (PEREIRA et al., 1997), com uma rica biodiversidade, tanto na fauna quanto na flora (SILVA; BATES, 2002; RATTER et al., 2003; LEITE et al., 2013). Algumas espécies arbóreas do Cerrado apresentam interesse comercial de sua madeira, frutos, sementes, fibras, resinas e óleos essenciais, entre outras partes (ALMEIDA et al., 1998), como o jatobá (Hymenea courbaril L.) e a copaíba (Copaifera langsdorffii Desf.) (LORENZI, 2002).

O jatobá é planta de porte arbóreo, pertencente à família Fabaceae, com denominações regionais como jataí, jutaí, entre outros, e apresenta boa adaptação a solos ácidos (NASCIMENTO et al., 2011). Os frutos são vagens indeiscentes, cilíndricas e lenhosas, medindo

\footnotetext{
${ }^{1}$ Recebido em 27.10.2012 aceito para publicação em 02.12.2013.

${ }^{2}$ Departamento de Agricultura/Fitotecnia, Universidade Federal de Lavras, UFLA, Brasil. E-mail: <arley.agro@yahoo.com.br> e <claubertmenezes@yahoo.com.br>.

${ }^{3}$ Departamento de Ciências Biológicas, Universidade Federal dos Vales do Jequitinhonha e Mucuri, UFVJM, Brasil. E-mail: <conceicao.santos@ufvjm.edu.br>.

${ }^{4}$ Departamento de Engenharia Florestal da Universidade Federal dos Vales do Jequitinhonha e Mucuri, UFVJM, Brasil. E-mail: <assisjr_ento@yahoo.com.br>,<drianogeraldo@yahoo.com.br>e <marcusasoares@yahoo.com.br>.
} 
de 8 a $15 \mathrm{~cm}$ de comprimento, com duas a seis sementes (De MELO et al., 2004; LORENZI et al., 2006). Sua polpa é farinácea e adocicada, com elevados teores de fibras, açúcares, proteínas, vitaminas e sais minerais, apresentando componentes nutricionais importantes para a saúde humana (ALMEIDA et al., 2008). O extrato da casca de jatobá contém terpenoides, compostos fenólicos e outras substâncias com ação antibactericida e de combate à fadiga, sendo utilizado como fitoterápico por seres humanos (SASAKI et al., 2009). Além disso, essa planta pode ser utilizada em recuperação de áreas degradadas, reflorestamentos e sistemas agroflorestais (NASCIMENTO et al., 2011).

A copaíba, ou “pau-d’óleo”, é uma Fabaceae, de ampla distribuição no Brasil, podendo ser encontrada, além do Cerrado, em mata de galeria e mata mesofítica de interflúvio. Produz óleo viscoso muito utilizado em indústrias farmacêuticas, cosméticas e de vernizes, corantes e lacas (ALMEIDA et al.,1998). A madeira é usada na construção civil e na fabricação de móveis e peças torneadas (LORENZI, 2002). Seus frutos são do tipo folículo, com uma semente preta envolvida por um arilo de cor laranja (VEIGA JÚNIOR; PINTO, 2002).

Muitas espécies da família Curculionidae são fitófagas na fase larval ou adulta, danificando diversas plantas (AMBROGI et al., 2009). Rhinochenus stigma Linnaeus, 1758 (Coleoptera: Curculionidae) pode danificar sementes de jatobá e copaíba, causando-lhes danos severos (COSTA LIMA, 1956; CRUZ et al., 2001; ALMEIDA et al., 2006). Essa espécie é encontrada na Amazônia, Nordeste e Sudeste brasileiro. Possui élitros amarelos com máculas vermelhas regulares, punções, estriais grossas e escamas de coloração branca nos élitros e rostro (WHITEHEAD, 1976). Seu ataque caracteriza-se pela raspagem do tegumento e perfuração do endosperma, com comportamento cletrofágico, ou seja, desenvolve-se no interior de sementes até a emergência do adulto.

Pupas fêmeas de besouros da família Curculionidae apresentam estrutura circular no final do abdome, com dois pontos no centro, comumente utilizada na sexagem nessa fase (SARRO et al., 2004; SOUZA et al., 2004). No entanto, em $R$. stigma essa estrutura não é parâmetro seguro para a distinção dos sexos por ser clara, dificultando sua visualização. Dessa forma, outros métodos de separação tornam-se necessários para uma distinção mais segura.

A morfometria e sexagem são importantes para a distinção e descrição de novas espécies de besouros curculionídeos. Entretanto, auxiliam no manejo integrado de insetos-praga, além de serem úteis em estudos de razão sexual, acasalamento e hábitos de oviposição desses insetos (SOUZA et al., 2004; SILVAFILHO et al., 2007). Este trabalho teve como objetivo avaliar a morfometria de pupas e adultos de R. stigma, bem como aspectos comportamentais, como a emissão de sons nos adultos, para auxiliar a sexagem dessa espécie.

\section{MATERIAL E MÉTODOS}

Pupas e adultos de R. stigma foram obtidos de 300 frutos de jatobá, coletados no Município de São Gonçalo do Rio Preto (1801’S 4322’W), Minas Gerais, Brasil, e levados ao Laboratório de Entomologia Florestal da Universidade Federal dos Vales do Jequitinhonha e do Mucuri (UFVJM) para sexagem.

As pupas foram separadas conforme a presença ou ausência de uma estrutura circular no final do abdome, com 37 indivíduos em cada grupo. Essas pupas foram submetidas à lavagem em água destilada e fixadas, individualmente, em recipientes de vidro de $50 \mathrm{~mL}$ com álcool 70\%, para melhor conservação de suas estruturas. Foram mensurados os seguintes caracteres morfométricos: comprimento do olho (partes inferior e superior), da antena (escapo, pedicelo/flagelo e total), do rostro e do corpo; e largura do rostro, do corpo e do final do abdome.

Os adultos de $R$. stigma foram acondicionados em potes plásticos transparentes de $500 \mathrm{~mL}$ e mantidos em sala em condições controladas $\left(25 \pm 2{ }^{\circ} \mathrm{C}, 60 \pm 10 \%\right.$ UR e fotoperíodo de $12 \mathrm{~h}$ ). Eles foram alimentados com polpa e sementes de jatobá e permaneceram no laboratório para observação da emissão de sons, características externas como a cor e pilosidade do exoesqueleto e posterior medição de estruturas morfológicas. A sexagem só foi possível, após a morte, pela exposição dos órgãos genitais (WHITEHEAD, 1976). Foram avaliados 23 indivíduos de cada sexo, sendo medidas as seguintes estruturas: comprimento do élitro (lateral e dorsal), pronoto (lateral e superior), rostro, antena (escrobo, escapo, pedicelo/flagelo), olhos e escutelo; e largura do élitro (base maior e base menor), base do pronoto e vértex (maior largura).

As avaliações das pupas e adultos foram feitas com o auxílio de microscópio estereoscópico, equipado com câmera de captura de imagem e programa Motic Images Plus 2.0, nos aumentos de 35x e 40x. Os dados 
foram submetidos ao teste $\mathrm{t}$ a $5 \%$ de probabilidade e analisados com o programa SAEG - Sistema para Análises Estatísticas, Versão 9.1, Universidade Federal de Viçosa (UFV) (SAEG, 2007).

\section{RESULTADOS}

As características morfométricas de pupas de $R$. stigma não foram satisfatórias para promover a diferenciação sexual, pois não houve diferenças significativas em nenhuma das variáveis analisadas (Tabela 1). No entanto, foram encontradas características morfológicas pouco evidentes nas pupas fêmeas de $R$. stigma como a presença, na parte ventral, de duas pontuações arredondadas em uma estrutura convexa circular, de coloração esbranquiçada no penúltimo urômero abdominal.

Foi evidente a dificuldade de observação do dimorfismo sexual, utilizando-se características morfológicas, em adultos de $R$. stigma devido à grande semelhança anatômica entre sexos (Figura 1A). Assim como nas pupas, não foram encontradas diferenças significativas nas variáveis mensuradas para os adultos (Tabela 2). Características como cor, pilosidade ou emissão de sons também não permitiram diferenciação segura dessa espécie. Para adultos de R. stigma, a sexagem só foi possível após a morte, pela dissecação dos órgãos genitais (Figura 1B).

\section{DISCUSSÃO}

O gênero Rhinochenus sp. possui várias espécies simpátricas de difícil distinção (WHITEHEAD, 1976). A não diferenciação do dimorfismo sexual em adultos de R. stigma pelas variáveis observadas, como forma, cor, dimensões das estruturas externas, aliada à não observação da emissão de sons nos adultos, pode ser indicativo de que a comunicação química, por meio de feromônios, seja efetiva nesta espécie.

A diferenciação sexual em Coleoptera Curculionidae é difícil de ser realizada na fase adulta, sendo necessário recorrer à dissecação e estudo das genitálias de machos e fêmeas (CASTAÑEDA-VILDÓZOLA et al., 2007). No entanto, características morfométricas e morfológicas das pupas podem ser eficientes para esse propósito (SARRO et al., 2004; SOUZA et al., 2004).

Embora pouco pronunciadas, as pontuações brancas no final do abdômen das pupas de $R$. stigma se assemelham à de pupas de outras espécies da família Curculionidae (BARRETO; ANJOS, 1999; SARRO et al., 2004; SOUZA et al., 2004) e são o único indicativo de sexagem disponível para esse estádio.

Fêmeas adultas de $R$. stigma apresentaram o primeiro esterno ligeiramente mais convexo e o abdômen um pouco mais largo, semelhante ao observado em outro trabalho (WHITEHEAD, 1976), porém essas características não são seguras para a sexagem. As condições nutricionais de Coleoptera podem influenciar as avaliações de morfometria. Adultos coletados no campo ou criados em laboratório podem apresentar diferenças nas dimensões das estruturas externas, como observado na broca do pedúnculo floral do coqueiro Homalinotus coriaceus (Coleoptera: Curculionidae) (SARRO et al., 2004).

Fêmeas adultas de Spermologus rufus e $H$. coriaceus (Coleoptera: Curculionidae) apresentaram corpo mais largo que os machos. No entanto, variações

Tabela 1 - Médias das estruturas de comprimento e largura da pupa de Rhinochenus stigma Linnaeus, 1758 (Coleoptera: Curculionidae).

Table 1 - Means of the structures of length and width of pupae Rhinochenus stigma Linnaeus, 1758 (Coleoptera: Curculionidae).

\begin{tabular}{lcc}
\hline Características morfométricas $(\mathrm{mm})$ & Macho* & Fêmea* \\
\hline Comprimento do olho inferior & $0,741 \pm 0,10$ & $0,728 \pm 0,10$ \\
Comprimento do olho superior & $1,287 \pm 0,11$ & $1,279 \pm 0,12$ \\
Comprimento da antena (escapo) & $0,768 \pm 0,11$ & $0,784 \pm 0,09$ \\
Comprimento da antena (pedicelo/flagelo) & $0,520 \pm 0,06$ & $0,518 \pm 0,06$ \\
Comprimento da antena & $1,288 \pm 0,14$ & $1,302 \pm 0,13$ \\
Comprimento do rostro & $1,328 \pm 0,12$ & $1,313 \pm 0,11$ \\
Comprimento do corpo & $7,968 \pm 0,59$ & $8,106 \pm 0,69$ \\
Largura do rostro & $0,658 \pm 0,10$ & $0,643 \pm 0,09$ \\
Largura do corpo & $4,224 \pm 0,36$ & $4,104 \pm 0,31$ \\
Largura do final abdome & $1,296 \pm 0,15$ & 0,8867 \\
\hline
\end{tabular}

*As médias não diferiram entre macho e fêmea, na linha, pelo teste $t$.

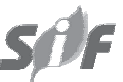

Revista Árvore, Viçosa-MG, v.38, n.1, p.25-30, 2014 
Tabela 2 - Medidas das estruturas do comprimento e largura de adultos de Rhinochenus stigma Linnaeus, 1758 (Coleoptera: Curculionidae).

Table 2 - Measures of length and width of the structures of adults Rhinochenus stigma Linnaeus, 1758 (Coleoptera: Curculionidae).

\begin{tabular}{lcc}
\hline Características morfométricas (mm) & Macho* & Fêmea* \\
\hline Comprimento lateral do élitro & $5,864 \pm 0,41$ & $5,883 \pm 0,44$ \\
Comprimento lateral do pronoto & $1,607 \pm 0,22$ & $1,608 \pm 0,12$ \\
Comprimento lateral do rostro & $1,451 \pm 0,17$ & $1,496 \pm 0,12$ \\
Comprimento do escrobo & $0,890 \pm 0,14$ & $0,888 \pm 0,12$ \\
Comprimento da antena (escapo) & $0,584 \pm 0,08$ & $0,607 \pm 0,05$ \\
Comprimento da antena (pedicelo/flagelo) & $1,288 \pm 0,07$ & $1,275 \pm 0,07$ \\
Comprimento dos olhos & $0,508 \pm 0,09$ & $0,575 \pm 0,12$ \\
Comprimento do escutelo & $0,297 \pm 0,06$ & $0,293 \pm 0,03$ \\
Comprimento do élitro dorsal & $5,692 \pm 0,51$ & $5,654 \pm 0,62$ \\
Comprimento superior do pronoto & $1,771 \pm 0,29$ & $1,797 \pm 0,25$ \\
Largura da base maior do élitro & $3,423 \pm 0,30$ & $3,477 \pm 0,18$ \\
Largura da base menor do élitro & $2,590 \pm 0,45$ & 0,4115 \\
Largura da base do pronoto & $1,497 \pm 0,22$ & 0,0247 \\
Largura do vértex (maior) & $2,432 \pm 0,11$ & 0,6055 \\
\hline
\end{tabular}

* As médias não diferiram entre macho e fêmea, na linha, pelo teste t.

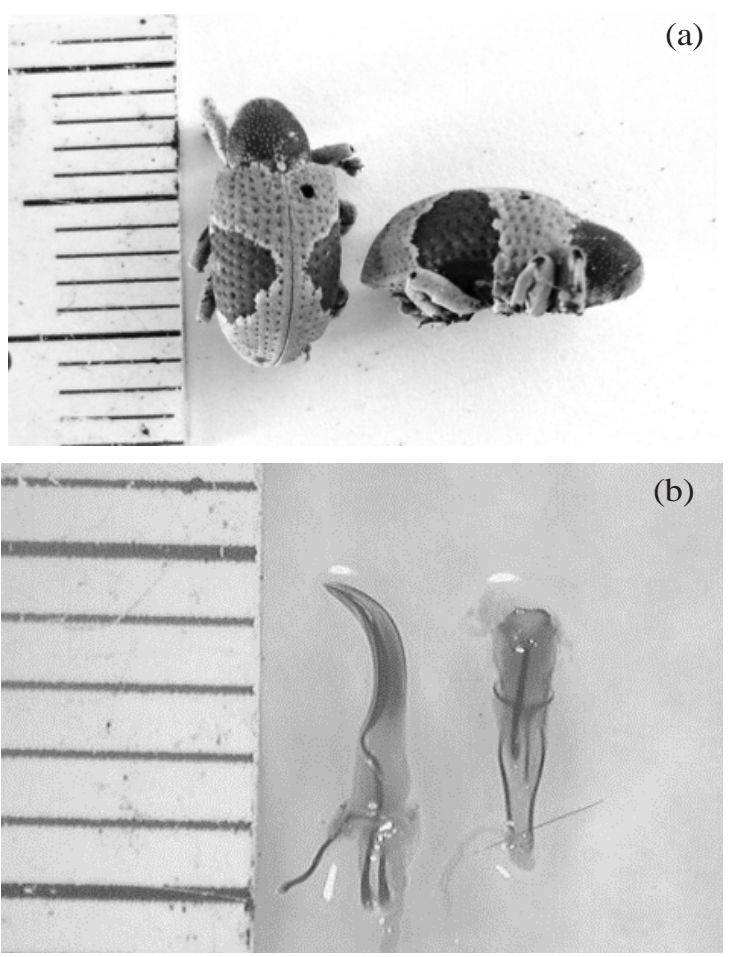

Figura 1 - Rhinochenus stigma Linnaeus, 1758 (Coleoptera: Curculionidae), macho (à esquerda) e fêmea (à direita) (A). Aparelho reprodutor de machos (à esquerda) e fêmeas (à direita) de $R$. stigma (B).

Figure 1 - Rhinochenus stigma Linnaeus, 1758 (Coleoptera: Curculionidae), male (left) and female (right) (A). Reproductive system of males (left) and female (right) of R. stigma (B). nessa característica a tornam inadequada para o reconhecimento dos sexos nessas espécies (SARRO et al., 2004). Entretanto, a sexagem de S. rufus pode ser determinada por meio do comprimento do rostro (BARRETO; ANJOS, 1999).

O tamanho, estruturas corporais como ângulos de inclinação, formato, posição de partes do corpo, manchas, pelos, espinhos e outras características têm sido bastante utilizadas na diferenciação sexual de Coleoptera, Curculionidae. Há grande similaridade entre os sexos de adultos da broca-da-batata-doce, Euscepes postfaciatus (Coleoptera: Curculionidae). No entanto, o metaesterno dos machos apresentou-se mais achatado longitudinalmente na área central, coberto densamente por escamas elipsoidais e semieretas, enquanto nas fêmeas esse éplano, com escamas mais esparsas (MENEZES, 2002). Em Rhinochenus maculipes (Coleoptera: Curculionidae), observou-se pouca quantidade de escamas cinzentas nos élitros das fêmeas em relação aos machos (WHITEHEAD, 1976). No gorgulho da flor do morango Anthonomus rubi (Coleoptera: Curculionidae), os espinhos nas coxas medianas de machos é a variável mais segura para a sexagem dessa espécie (INNOCENZI et al., 2002). Fêmeas adultas de $H$. coriaceus e Conotrachelus psidii (Coleoptera: Curculionidae) possuem o penúltimo tergito abdominal, projetando-se sobre o último (SARRO et al., 2004).

A comunicação dos insetos em geral pode ser visual, sonora ou química. Para ocorrer de maneira efetiva, é necessário que as informações sejam passadas de 
forma eficiente entre o emissor e o receptor. A grande semelhança entre os sexos em Coleoptera: Curculionidae pode ser indicativo de que a comunicação visual seja pouco eficiente nessa família. Emissões sonoras são mais efetivas entre algumas espécies desses insetos, envolvendo a estridulação emitida por um dos sexos, como ocorre em machos de Heilipus odoratus e $C$. psidii (Coleoptera: Curculionidae) (SILVA FILHO et al., 2007; NUNES et al., 2009). A comunicação química é importante entre os insetos, com destaque para os feromônios, que são substâncias voláteis envolvidas nas interações intraespecíficas (SYMONDS; ELGAR, 2008). Embora não seja sexual, o feromônio da brocado-olho-do-coqueiro Rhynchophorus palmarum (Coleoptera: Curculionidae) já foi bastante estudado e está disponível comercialmente, com o nome técnico de Rincoforol. Trata-se de um feromônio de agregação, cuja função é, normalmente, indicar a presença de alimento, promovendo a proximidade dos indivíduos, o que facilita o encontro dos casais para reprodução (NAVARRO et al., 2002).

\section{CONCLUSÃO}

Pupas e adultos de $R$. stigma não apresentaram diferenças significativas nas variáveis mensuradas, não sendo possível utilizar a morfometria para diferenciação dos sexos nesses estádios. Não foram observadas diferenças na forma, cor ou pilosidade do corpo, nem emissão de sons pelos adultos dessa espécie que pudessem auxiliar a sexagem. Para adultos de $R$. stigma, a separação dos sexos só é possível, após a morte, pela dissecação dos órgãos genitais. O comportamento e a comunicação de $R$. stigma devem ser mais bem explorados, com a finalidade de conhecimento de sua biologia e ecologia. Além de permitir a definição de possíveis métodos de captura e criação em laboratório, servem como estratégia de estudo para métodos de manejo integrado dessa praga, devendo ser objetivos de novos estudos.

\section{AGRADECIMENTOS}

Ao Conselho Nacional de Desenvolvimento Científico e Tecnólogico (CNPq), à Coordenação de Aperfeiçoamento de Pessoal de Nível Superior (CAPES) e à Fundação de Amparo à Pesquisa do Estado de Minas Gerais (FAPEMIG), pelo apoio financeiro; e ao Departamento de Entomologia da Universidade Federal de Viçosa (UFV).

\section{REFERÊNCIAS}

ALMEIDA, S.P. et al. Cerrado: espécies vegetais úteis. Planaltina: EMBRAPA - CPAC, 1998. 464p.

ALMEIDA, C.I.M.; LEITE, G.L.D.; ROCHA, S.L.; MACHADO, M.M.L.; MALDONADO, W.C.H. Fenologia e artrópodes de Copaifera langsdorffii Desf. no cerrado. Revista Brasileira de Plantas Medicinais, v.8, p.64-70, 2006.

ALMEIDA, S.P. et al. Frutas nativas do cerrado: caracterização físico-química e fonte potencial de nutrientes. In: SANO, S.M.; ALMEIDA, S.P.; RIBEIRO, J.F. Cerrado: ecologia e flora. Brasília, DF: EMBRAPA Informação Tecnológica, p.353-381, 2008.

AMBROGI, B.G.; VIDAL, D.M.; ZARBIN, P.H. G.; ROSADO-NETO, G.H. Feromônios de agregação em Curculionidae (Insecta: Coleoptera) e sua implicação taxonômica. Química Nova, v.32, p. 2151-2158, 2009.

BARRETO, M.R.; ANJOS, N. Spermologus rufus Boheman 1843 (Coleoptera: Curculionidae). Ciência e Agrotécnica, v.23, p.358-364, 1999.

CASTAÑEDA-VILDÓZOLA, A. et al. Genitalia de tres especies de Heilipus Germar (Coleoptera:

Curculionidae) que dañan frutos de aguacate (Persea americana Mill) en México y Costa Rica.

Neotropical Entomology, v.36, p.914-918, 2007.

COSTA LIMA, A. M. Insetos do Brasil.

(Coleópteros) Rio de Janeiro. Escola Nacional de Agronomia, 1956. 373p Tomo 10. (Série Didática, 12.)

CRUZ, E. D.; MARTINS, F.O.; CARVALHO J.E.U. Biometria de frutos e sementes e germinação de jatobá-curuba (Hymenaea intermedia Ducke, Leguminosae - Caesalpinioideae). Revista Brasileira de Botânica, v.24, p.161-165, 2001.

DE MELO, M.G.G.; MENDONÇA, M.S.; MENDES, A.M.S. Análise morfológica de sementes, germinação e plântulas de jatobá (Hymenaea intermedia Ducke var. adenotricha (Ducke) Lee \& Lang.) (Leguminosae Caesalpinioideae). ACTA Amazônica, v.34, p.9-14, 2004.

Revista Árvore, Viçosa-MG, v.38, n.1, p.25-30, 2014 
INNOCENZI, P.J. et al. Sexing adults of the strawberry blossom weevil Anthonomus rubi (Coleoptera: Curculionidae). Journal Applied Entomology and Zoology, v.126, p.159-160, 2002.

LEITE, G.L.D. et al.. Seasonal abundance of galling insects (Hymenoptera) on Caryocar brasiliense (Malpighiales: Caryocaraceae) trees in the cerrado. Florida Entomologist, v.96, p.797-809, 2013.

LORENZI, H. Árvores brasileiras: manual de identificação e cultivo de plantas arbóreas nativas do Brasil. 2.ed. Nova Odessa: Plantarum, 2002. v.2. p.331.

LORENZI, H. et al. Frutas brasileiras e exóticas cultivadas: de consumo in natura. Nova Odessa: Instituto Plantarum, 2006.

MENEZES, E. L. A. A broca da batata-doce (Euscepes postfasciatus): descrição, bionomia e controle. Seropédica: Embrapa, 2002. p.12. (Circular Técnica, 6).

MENEZES, E.L.A. A Broca da Batata-Doce (Euscepes postfasciatus): Descrição, Bionomia e Controle. EMBRAPA, Circular Técnica $n^{\circ}$ 6, p.12, 2002.

NASCIMENTO, H.H.C. et al. Análise do crescimento de mudas de jatobá (Hymenaea courbaril L.) em diferentes níveis de água no solo. Revista Árvore, v.35, p. 617-626, 2011.

NAVARRO, D.M.A.F. et al. Aspectos práticos relacionados ao uso do Rincoforol, o feromônio de agregação da Broca-do-olho-do-coqueiro Rhynchophorus palmarum L. (Coleoptera: Curculionidae) no controle de pragas do coqueiro. Análise de sua eficiência em campo. Química Nova, v.25, p.32-36, 2002.

NUNES, A.B.; RONCHI-TELES, B.; SPIRONELLO, W. Comportamento de estridulação em Heilipus odoratus Vanin \& Gaiger (Coleoptera, Curculionidae, Molytinae). Revista Brasileira de Entomologia v.53, p.334-336, 2009.

PEREIRA, G. et al. Area and population of the Brazilian savanna. Pesquisa Agropecuária Brasileira, v.32, p.759-763, 1997.

Revista Árvore, Viçosa-MG, v.38, n.1, p.25-30, 2014
RATTER, J.; BRIDGEWATER, S.; RIBEIRO, J.F. Analysis of the floristic composition of the Brazilian Cerrado vegetation. III: comparison of the woody vegetation of 376 areas. Edinburgh Journal of Botany, v.60, p.57-109, 2003.

\section{SAEG. Sistema para Análises}

Estatísticas, Versão 9.1. Viçosa, MG: Fundação Arthur Bernardes/UFV, 2007.

SARRO, F.B.; CROCOMO, W.B.; FERREIRA, J.M.S. Aspectos da biologia e morfologia da broca do pedúnculo floral do coqueiro, Homalinotus coriaceus (Gyllenhal) (Coleoptera: Curculionidae). Neotropical Entomology, v.33, p.7-12, 2004.

SASAKI, K. et al. High-Performance Liquid Chromatographic Purification of oligomeric procyanidins trimers up to nonamers, derived from bark of jatobá (Hymenaea courbaril).

Bioscience, Biotechnology and Biochemistry, v.73, p.1274-1279, 2009.

SILVA, J.M.C.; BATES, J.M. Biogeographic patterns and conservation in the South American Cerrado: a tropical savanna hotspot.

BioScience, v.52, p.225-233, 2002.

SILVA-FILHO, G.; BAILEZ, O.E.; VIANA-BAILEZ, A.M. Dimorfismo sexual do gorgulho-da-goiaba Conotrachelus psidii Marshall (Coleoptera: Curculionidae). Neotropical Entomology, v.36, p.520-524, 2007.

SOUZA, W.O. et al. Description of the larva and pupa of the papaw borer weevil Pseudopiazurus papayanus (Marshall) (Coleoptera, Curculionidae, Piazurini).

Revista Brasileira de Entomologia, v.48, p.331-334, 2004.

SYMONDS, M.; ELGAR, M. The Evolution of Pherormone Diversity. Trends in Ecology \& Evolution, v.23, p.220-228, 2008.

VEIGA JUNIOR, V.F.; PINTO, A.C. O gênero Copaifera L. Química Nova, v.25, p.273-286, 2002.

WHITEHEAD, D.R. Classification and evolution of Rhinochenus Lucas (Coleoptera: Curculionideae: Cryptorhynchineae), and quaternary middle american zoogeography. Questiones Entomologicae v.12, p.118-201, 1976. 